\title{
Efficient-Path Selection Scheme Using Optimized Adhoc on Demand Multipath Routing Protocol For Adhoc Networks
}

\author{
Bekan Kitaw \\ Faculty of Computing, Jimma Institute of Technology, Jimma University, Jimma, Ethiopia \\ Email: bekankitawm@gmail.com \\ Fisseha Bayu \\ Faculty of Computing, Jimma Institute of Technology, Jimma University, Jimma, Ethiopia \\ Email: fisseha_bayu@yahoo.com \\ Gemechu Birhanu \\ Faculty of Computing, Jimma Institute of Technology, Jimma University, Jimma, Ethiopia \\ Email: g4rfsh@gmail.com
}

\begin{abstract}
Mobile Adhoc Networks are used in various areas each of which has its own set of requirements. In this paper we concentrate on application areas which require fast data delivery, less delay and high availability of the network. Fast delivery and less delay concepts are related to the relative position and status of nodes (links) while network availability is a direct cost of overall network lifetime. Our focus here is on developing a network layer strategy, i.e. one that uses routing protocols. For MANET, in some applications packets become useless as a result of delay and distance between nodes. Hence it is required to design Efficient-Path selection scheme using optimized ondemand Multipath routing protocol with goals of improving throughput, delay and data delivery. With this our new approach we are going to evaluate the performance of our protocol against the traditional AOMDV. The practical simulation is done using NS2 network simulator.
\end{abstract}

Keywords: AOMDV, Multipath, MANET, NS2, routing, throughput.

\section{[1] INTRODUCTION}

$\mathrm{W}_{\mathrm{it}}$ he advent of modern technology, every one desires to stay connected all over the world. Since telephonic conversation is no longer the only requirement for telecommunication, users like to share videos, play interactive games, hold video conferences and use other multimedia applications. Apart from this they own more than one communication devices. Thus there is a need for inter-connectivity [1] which can be through: infrastructure and Adhoc mode as shown in Figure 1 below. Adhoc mode is used to connect wireless clients directly together, without the need for router or AP [2] [17]. There are various routing protocols that are designed for Adhoc network among which AOMDV [3] is one of them with Multipath reactive, Link disjoint and node disjoint paths.
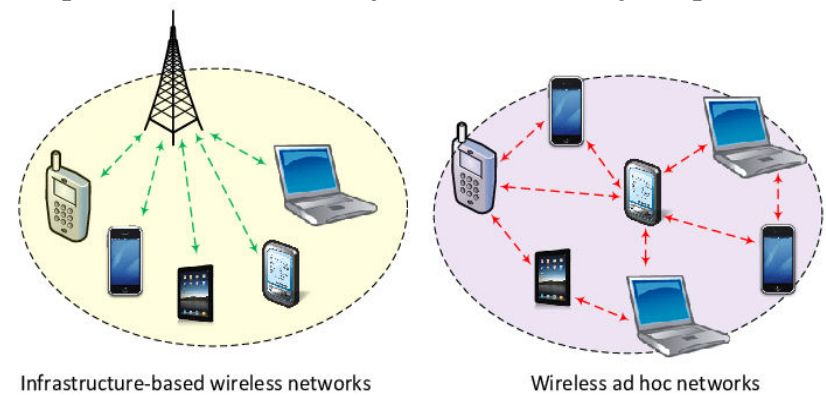

Fig 1: Infrastructure Vs Infrastructure-less networks [22]
There is a growing desire for wireless networks to support real time multimedia applications such as video conference, voice over IP (VoIP) and emergency application like medical alert system which require the network to provide guarantees on some form of Quality of Service (QoS). QoS is the ability of the network to provide some level of assurance for consistent network data delivery [4] [5]. Apart from QoS the network guarantees on the distrust avoidance so that they behave better QoS. The network is expected to guarantee set of measurable pre-specified service attributes to the users in terms of delay, throughput, probability of packet loss, etc.

Many protocols were developed to alleviate problems in routing; however, none of them could completely overcome the challenges faced in MANET i.e. they converge fast to a single solution that decreases the network performance. Most of them contain tunable parameters and methods to automate the selection of optimal routes for different network conditions. However, the algorithm does not contain the integration of several parameters for efficient network. Hence, providing efficient path selection scheme in MANET is hard to guarantee. There are generally no strong guarantees of timely delivery, and hence delay sensitive data may also deliver late as the cases with medical conditioning alert which if late may result in the death of the patient. Further analysis of the different routing protocols and the way in 
which they attempt to improve QoS of network traffic was stated by various researchers. Since some paths may experience large delay because of several reasons and some nodes even move away from their neighbor node which indirectly result in large delay and less packet delivery.

In this research, we are trying to focus on improving the performance of traditional AOMDV routing protocol using optimized route selection mechanism. This should be achieved through by providing support for these QoS requirements in this protocol. Our main research question therefore is: "Can we improve AOMDV to guarantee data delivery so as it would be capable for use in an emergency application of Adhoc Networks?"

The remaining sections are organized as follows. Section 2 introduces related works which are more related to our work. Section 3 presents the design and description of the proposed work, model and algorithms. Section 4 provides an extensive simulation study and evaluation of the proposed algorithms. Finally, the conclusions of the research and recommendations of future works are presented in Section 5.

\section{[2] RELATED WORK}

In MANET Selection of one route from multiple routes is a critical issue. Various researchers use different metrics for selection of the route as part of their criteria. But due to various reasons this selection is still not efficient in some scenarios which we are going to deal in this paper.

In [6] protocol with enhanced route discovery mechanism that avoids congestion in the route is proposed. The proposed protocol, Novel Load Balancing Scheme for Multipath Routing, selects route on the basis of traffic load on the node and resets path as the topology changes. Instead of transmitting entire data through one route, new efficient paths are discovered from time to time during transmission and this will balance the load on a node and extends the lifetime of the node. Since originally the number of links passing over a node is not restricted, which would result in high delay, more control overheads and performance degradation. The paper introduces a threshold value that limits the number of links passing over a node. A counter indicating the number of links or paths passing through a given node will incremented by one for every new request and the threshold value is compared to see whether the maximum number of connections has been reached or not. According to this study, the term load metric reflects how busily a node is engaged in receiving and forwarding packets over the wireless media.

The author in [7] proposes a new protocol called Bandwidth-aware (BW-AOMDV) protocol. The paper proposes a solution to utilize available bandwidth of the channel for on demand multiple disjoint paths. The approximate bandwidth of a node is used to find the available bandwidth of the path. The routing table entry structure of AOMDV is modified for the proposed method in which only one field is added which gives the information about the path bandwidth of the multiple paths stored in route list entries. The source chooses the primary route for data forwarding on the basis of path bandwidth. According to the paper, the source is able to learn the bandwidth of the multiple paths during the route discovery by using the Maximum-Minimum approach to measure the quality of the path. The bandwidth of the route is determined periodically in order to find the optimal route in the network topology with the help of detector packet. Even though the channel bandwidth is utilized in order to improve the network performance, still there is no guarantee for data delivery.

An approach to improve the energy efficiency by using an Energy Efficient Routing for MANET using on demand Multipath routing protocol is discussed [8]. The paper mainly focuses on routing mechanisms that reduces the total energy consumption so that to increase the network lifetime of MANETs. It mainly considers transmission power and residual energy of the nodes. The proposed energy efficient routing protocol for MANETS tries to reduce energy consumption by means of an energy efficient routing metric, used in routing table computation instead of the minimum hop metric. This way, a routing protocol can introduce energy efficiency while selection of routes.

As a result of some limitations with energy aware routing mechanisms, [9] paper introduced energy efficient and bandwidth aware routing in which the source selects the primary route for data forwarding on the basis of minimal residual energy and available bandwidth for on demand multiple disjoint paths and so that to improve QoS. The main goal is to find energy efficient and maximum available bandwidth shortest path between sources to destination. They reduce the energy consumption, bandwidth aware average end-to-end delay, routing overhead and normalized routing overhead. They also improve packet delivery ratio and throughput as compared to the original AOMDV protocol.

In [11] proposed a model for Load Aware and Energy Efficient Approach to Improve Network Connectivity in MANET with the goals of improving throughput as well as energy efficiency. Since in the original AOMDV inefficient load balancing technique resulted into increasing routing overhead, poor packet delivery ratio and other parameters, this paper proposed novel approach towards this problem. Providing supports for QoS and extended network lifetime concurrently, the paper has two main characteristics: method of link estimation proposed for energy efficiency improvement and another is learning of load balancing in order to achieve the improved QoS solutions. But this protocol is failed to evaluate delay between each nodes and end to end delay along a given path for the route metrics.

In [12], a new enhanced routing protocol has been designed for path selection depending on two main 
metrics: node energy factor and node distance from its transmitting predecessor node. According to the assumption node energy factor has been used as a metric to prolong life time of selected route, while the distance is being used as a metric to obtain stable route with relatively minimum hop counts in addition to minimizing routing control packets. Even though the paper minimizes the rebroadcasting process and obtain energy efficient stable route, still there might be some nodes which seems trusted i.e. having much energy and nearest to the predecessor node than other but are misbehavior in that they experiences large delay.

Going through all the previous work listed, we observed that still there is no guarantee for packet delivery esp. in delay sensitive applications. Thus we tried to improve the performance of MANET for emergency applications by integrating: node location (for distance computation), Packet travel time, energy factor and hop count using optimized route selection mechanism.

\section{[3] PROPOSED SOLUTION}

Even-though many researchers consider various metrics for route selection still there is no guarantee for the packet delivery especially in applications in which delays might cause packet to be useless as in an emergency application. Our proposed approaches do consider multiple metrics approach for the route selection guaranteeing packet delivery for this kind of application. Thus, we tried to use a combined metrics approach which considers Packet travel time, Position of node for distance computation, node energy factor and the hop count metrics. Combining this all would result in efficient and effective path selection and that is why we call our newly proposed scheme EP-AOMDV protocol.

Our new Scheme mainly focuses on the third layer of MANET protocol stack, the Network layer, where various Adhoc routing protocols found. We specifically integrate our new algorithm into AOMDV Adhoc routing protocol.

EP-AOMDV chooses the efficient path among all the potential paths by our newly proposed algorithm, where we make the following two changes:

- Source node places a time-stamp for packet travel time estimation, (X, Y) coordinate position of node for distance estimation and its own node energy factor on the RREQ packet when it originates the route discovery using RREQ.

That is, as indicated in Table 1 we modify the RREQ packet of AOMDV to include Packet travel time, Distance and Node energy factor.
Table1: RREQ packet structure of EP-AOMDV

\begin{tabular}{|c|c|c|}
\hline Type & Last hop & Hop count \\
\hline \multicolumn{3}{|c|}{ RREQ id } \\
\hline \multicolumn{3}{|c|}{ Destination IP Address } \\
\hline \multicolumn{3}{|c|}{ Destination Sequence Number } \\
\hline \multicolumn{3}{|c|}{ Source IP Address } \\
\hline \multicolumn{3}{|c|}{ Source Sequence Number } \\
\hline \multicolumn{3}{|c|}{ Packet Travel Time } \\
\hline \multicolumn{3}{|c|}{ Distance } \\
\hline \multicolumn{3}{|c|}{ Node Energy Factor } \\
\hline
\end{tabular}

- We modify the routing table as shown below by adding a new field to record the minimum energy and the combination of packet travel time (PTT), distance and hop count for weight factor of the path calculation.

Table2: Routing table entry structure of EP-AOMDV

\begin{tabular}{|c|c|c|c|}
\hline $\begin{array}{l}\text { Dest } \\
\text { IP }\end{array}$ & $\begin{array}{l}\text { Sequen } \\
\text { num. }\end{array}$ & $\begin{array}{l}\text { Advertised } \\
\text { Hcount }\end{array}$ & 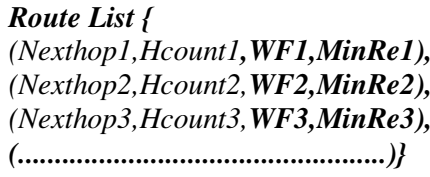 \\
\hline
\end{tabular}

Each receiving node will calculate distance and minimum node energy factor when a RREQ is received and will look up the time-stamp of the RREQ packet. According to our assumption it is the function of destination node to calculate the packet travel time. In Adhoc networks, Packet travel time also called the transmission time is the time it takes for a packet to deliver from source $S$ to destination $\mathrm{D}$ and thus it is calculated by $\mathrm{D}$ unlike the round trip time which is the time from the start of the transmission until a response (ACK) is received at the same node. The destination node will calculate the packet travel time [13] using the following equation:

$\mathrm{PTT}=\mathrm{CT}-\mathrm{OT}$

Where, PTT is the Packet Travel Time

CT is the Current Time Stamp

OT is the Packet origination Time Stamp

In addition to this, each receiving node will calculate the distance [14] [15] between itself and the predecessor node when it receives RREQ packet.

$d=\sqrt{\left(X_{2}-X_{1}\right)^{2}+\left(Y_{2}-Y_{1}\right)^{2}}$

Where $\left(\mathrm{X}_{1}, \mathrm{Y}_{1}\right)$ and $\left(\mathrm{X}_{2}, \mathrm{Y}_{2}\right)$ are the coordinates of RREQ transmitting and receiving node respectively. Here the distance between each and every individual neighbor node is calculated and then this value will be added with their corresponding precedence values (i.e. the distance that come with the RREQ packet) when the RREQ packet is sent.

In our EP-AOMDV, it is assumed that all nodes have same transmission range $(\mathrm{R})$, initial energy $\left(\mathrm{E}_{\mathrm{i}}\right)$, and are at their 
own ( $\mathrm{x}, \mathrm{y})$ coordinates. Accordingly, a node can be selected as next hop in route between source and destination if and only if it has energy above declared threshold value and then the other metrics are considered along the path. For each intermediate node in a disjoint path, the residual energy and the nodes energy factor is calculated using equation (3) below and minimum of all is stored in the RREQ packet.

$$
N E F=\frac{E_{i}}{E_{r}}
$$

Where, $\mathrm{E}_{\mathrm{i}}$ : Node initial energy

$\mathrm{E}_{\mathrm{r}}$ : Node residual energy

NEF: Node energy factor

Then after calculating this, we use a weight-based routing scheme, where a weight is assigned for each metric based up on their priority level [13] since our protocol is for emergency application. Nodes update their routing tables according to the path costs computed using the nodes weights received at each time period. The metrics are normalized and they contribute additively to the nodes weight computation with some multiplicative factors, as shown in equation (4). So in our protocol, WF value is assigned to each path using the following general linear equation:

$W F_{(p)}=\sum_{i=1}^{n} a_{i} m$

Where WF is the value assigned to the path. i runs for all $n$ number of metrics, and $a_{i}$ is the weight assigned to metric m.

Here in our protocol, we combine three metrics (Packet Travel Time, distance, and Hop count) with different weights to get WF value of a node as:

$W F\langle\ldots . P\rangle=W 1 * P T T\langle P\rangle+W 2 * D\langle P\rangle+W 3 * H C\langle P\rangle$.

Where, WF (P) is a weight factor,

PTT $(\mathrm{P})$ packet travel time of path $\mathrm{P}$,

D (P) Distance of path from Source $S$ to $D$ destination, HC (P) Total hop count of path P. And the weights $\mathrm{W} 1, \mathrm{~W} 2$ and $\mathrm{W} 3$ are chosen based on the network dynamics and application requirements.

In this study, since we assumed to develop the protocol for emergency application, we assign the weight values based upon their priority level [13]. Thus, in routing selection process we uses 0.5 for $\mathrm{W} 10.3$ for $\mathrm{W} 2$ and 0.2 for $\mathrm{W} 3$ such that $\mathrm{W} 1+\mathrm{W} 2+\mathrm{W} 3=1$ condition is satisfied. That is $0.5+0.3+0.2=1$ consequently, the sum of the objectives has to be minimized for the path to be efficient and this minimum weight Factor (MWF) can be computed by:

$$
\mathrm{MWF}=\min (\mathrm{WF}\langle\mathrm{P} 1\rangle, \mathrm{WF}\langle\mathrm{P} 2\rangle \ldots, \mathrm{WF}\langle\mathrm{Pn}\rangle) \ldots
$$

The path with MWF value is selected as a best path for data transmission. When the destination node responds to a RREQ for the first time with a RREP, the destination shall store the WF and minimum remaining energy factor MinRe value in the routing table entry along with other entries. Similarly intermediate nodes will add reverse path entries with calculated WF and minimum remaining energy factor MinRe values when they receive RREQs.

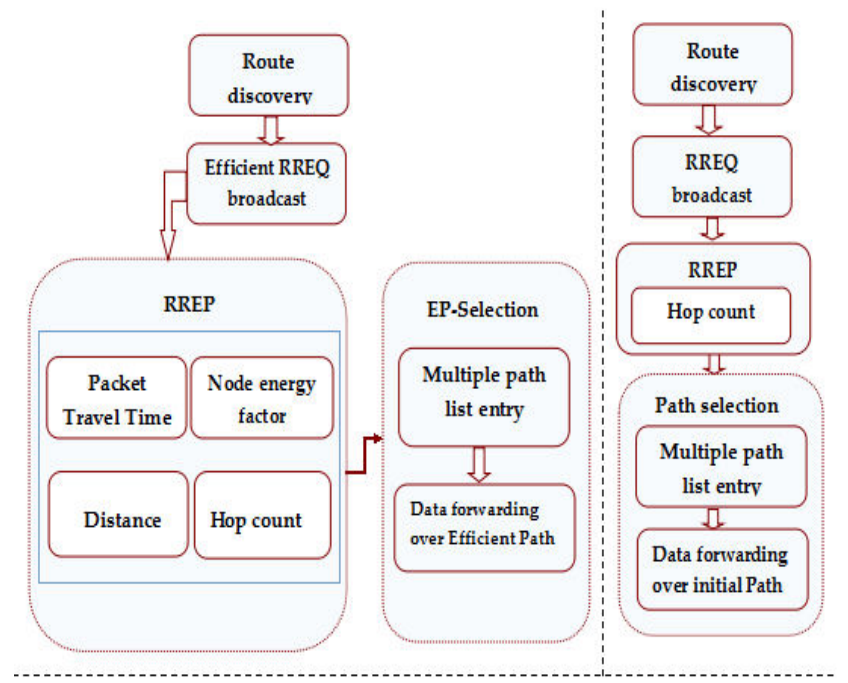

Fig 2: Block diagram of EP-AOMDV and AOMDV

As indicated in the above Figure 2, our proposed approach selects the best path based on the combined metrics unlike that of the original AOMDV which selects path based on hop count metrics only. The RREP contains the combination of packet travel time, node energy factor, distance and the hop count metrics in our scheme as shown in the block diagram. Here in figure shown below we try to represent the diagrammatical work flow of our proposed scheme starting from the RREQ initialization to route update procedure.

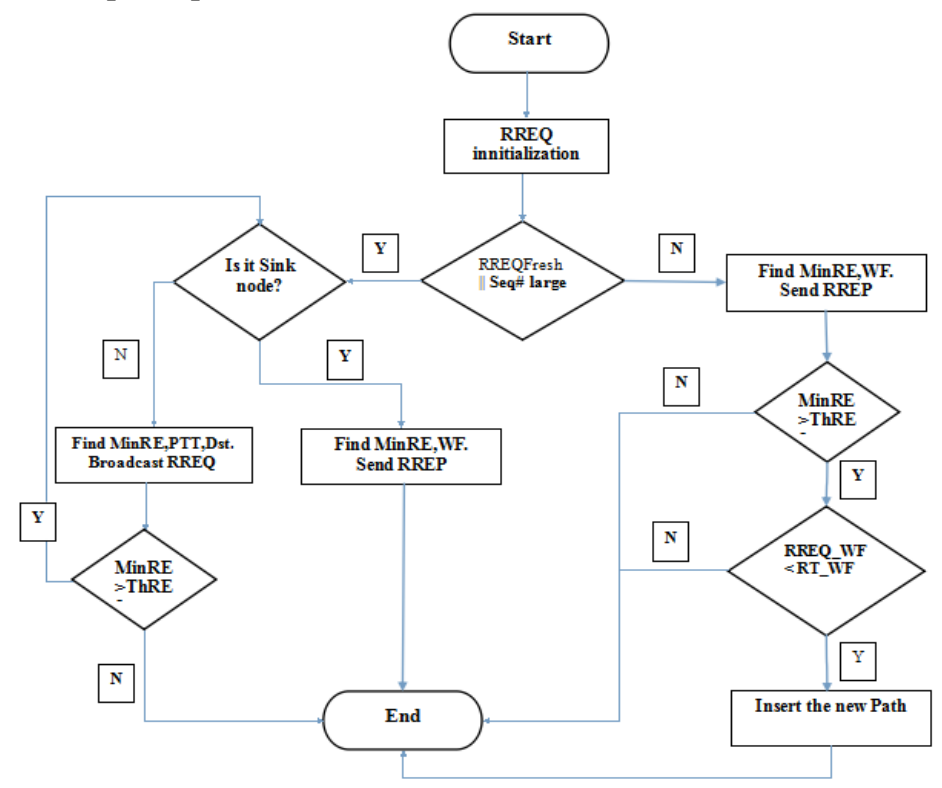

Fig 3: Flow chart Work flow of EPAOMDV Protocol

Here the following Algorithm shows the proposed algorithm in selection of efficient path and the route update rules of the newly proposed algorithm for MANET ad-hoc network with $n$ nodes, out of which two of them 
are source and destination. Each node $\mathrm{n}$ maintains its own $(\mathrm{x}, \mathrm{y})$ coordinate, initial energy and transmission range.
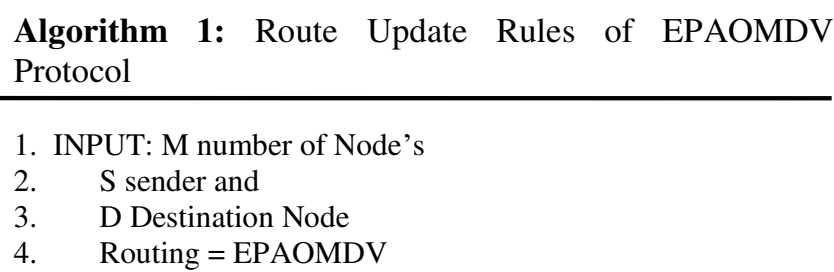

5. OUTPUT: Efficient path selection with less delay and maximum throughput guaranteeing data delivery.

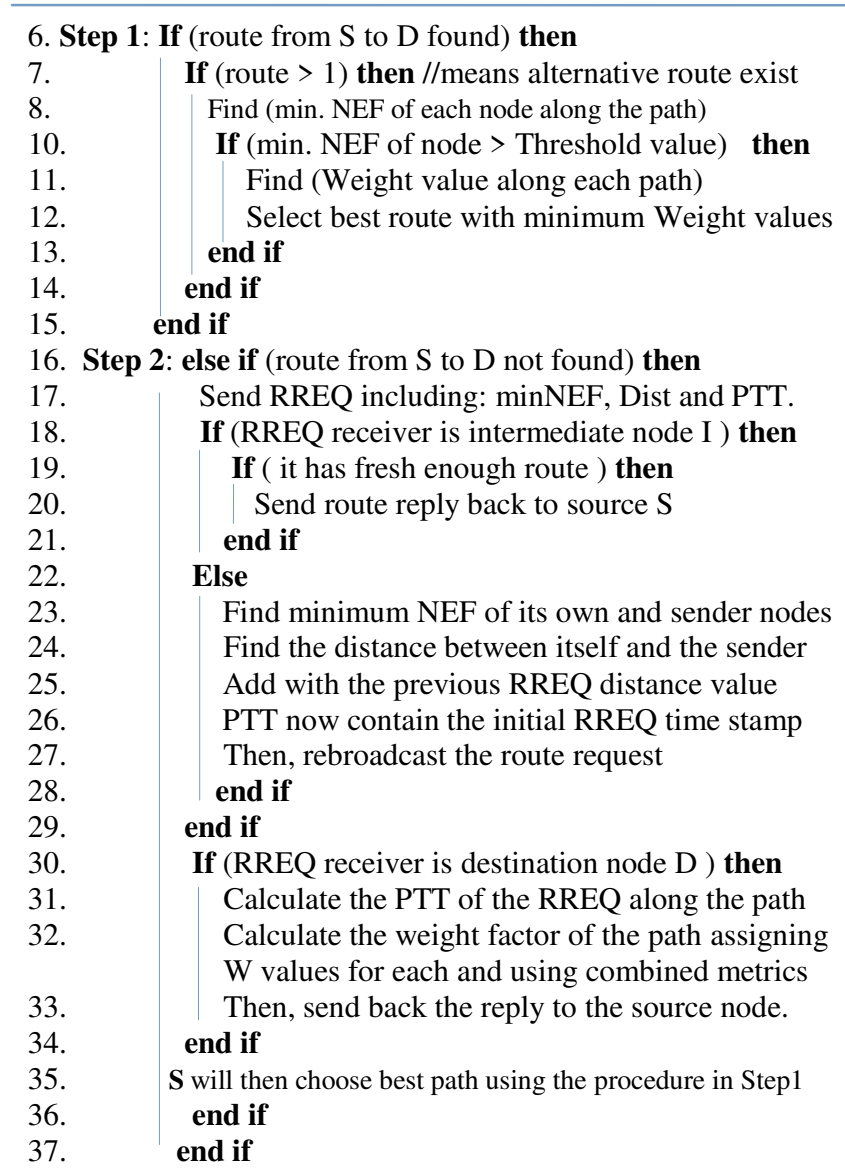

\section{[4] INVESTIGATION OF SCENARIO}

We tried to validate the working scenario for our proposed routing protocol. As shown in the following diagram, we assign a certain default values for every sensor nodes so that they let us to differentiate the effect of each metrics that we used in our path selection.

As the scenario in Figure 4 indicates, there are 8 intermediate sensor nodes, one source and one destination nodes. The packet travel time (PTT), Distance (Dist), and Node energy factor (NEF) values are given to validate our assumption which is described next.

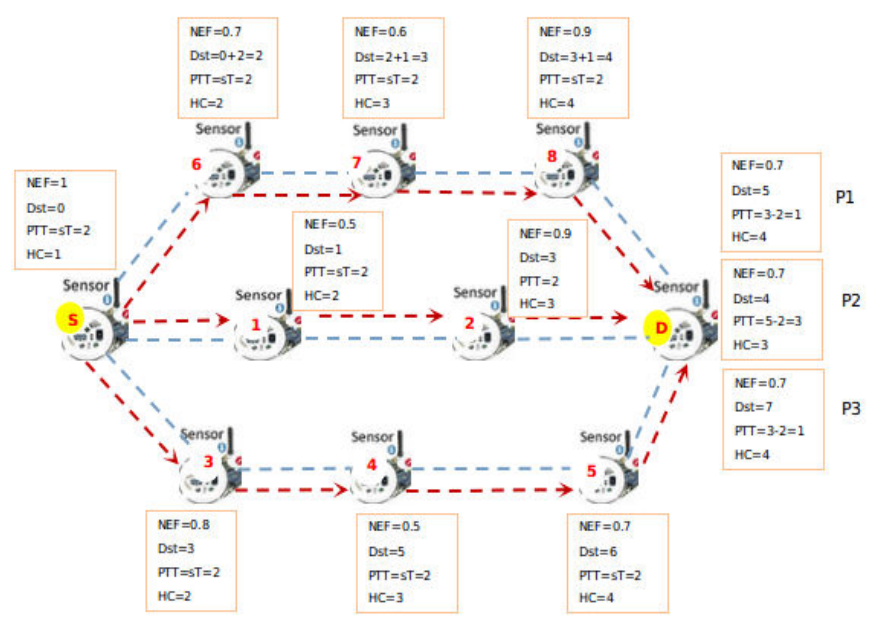

Fig 4: Scenario illustration of EPAOMDV Protocol

Note that, on the above scenario the packet travel time is calculated using the delivery time stamp of 3 in path $\mathrm{P} 1,5$ in path $\mathrm{P} 2,3$ in path $\mathrm{P} 3$ respectively and also the sent time of RREQ is assigned to be 2 since in all the three paths packet is sent at the same time. As shown above we have three different paths for the packets to be sent from source $\mathrm{S}$ to destination D. These three paths are:
1. $\mathrm{S} \rightarrow 6 \rightarrow 7 \rightarrow 8 \rightarrow \mathrm{D}$
2. $\mathrm{S} \rightarrow \mathbf{1} \rightarrow \mathbf{2} \rightarrow \mathrm{D}$
3. $\mathrm{S} \rightarrow \mathbf{3} \rightarrow \mathbf{4} \rightarrow \mathbf{5} \rightarrow \mathrm{D}$

In which the number of hop count is 4,3 and 4 respectively. Using the original AOMDV protocol the second path with minimum hop count will be chosen as the best path. But it has large delay in that the packet travel time is large through this path.

For an investigation purpose we assumed the working scenario of biosensor Ad hoc network that consists of numerous biosensors in which the network consists of a patient as a source node $\mathrm{S}$, other intermediate nodes (in our case node 1-8) and an information center that is destination node, D. Here the patient is equipped with a medical sensor network on his body. There is a gateway connected to the sensor network which is used to periodically collect health and medical data from the sensors in sensor network carried by the patient. The data collected by the gateway is sent to the destination, the doctor in our case using an Adhoc network. So if the data is Delay sensitive Data Type (e.g. a life- threatening medical condition alert such as high blood pressure), immediate care should be given for the patient so as to save his/her life. Thus, efficient path with less delay and maximum throughput must be chosen in delivering those data from source node to destination node.

Accordingly for the above scenario described in Figure 4 implementing our approach we can find an efficient path. That is, finding the weight value for all paths:

$$
\begin{aligned}
\mathrm{WF}(\mathrm{P} 1) & =0.5 * \mathrm{PTT}+0.3 * \mathrm{Dst}+0.2 * \mathrm{HC} \\
& =0.5 * 1+0.3 * 5+0.2 * 4 \\
& =2.8
\end{aligned}
$$




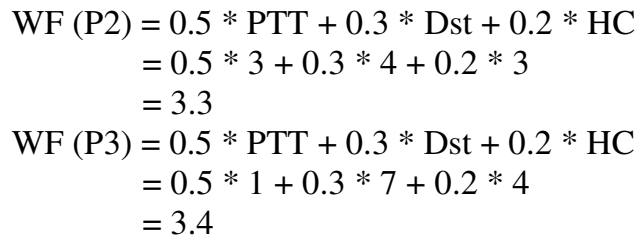

Since in our proposed approach the path with minimum weight factor will be chosen as the best path, path P1 will be chosen as best path resulting in less delay and guaranteeing data delivery.

\section{[5] SIMULATIONS AND RESULT ANALYSIS}

The performance analysis of proposed routing protocols can be done by means of designing and deploying real MANET environments. But due to various limitations such as accessibility of sensor nodes and time limitations we designed our MANET topology by means of simulation. To test our proposed EP-AOMDV routing protocol for MANET network, we have used NS2 network simulator [20] [21] and performed a simulation experiment using various evaluation metrics/parameters.

We simulate both efficient path AOMDV (our proposed protocol i.e. EPAOMDV) and the original AOMDV protocol. Accordingly, the nodes are deployed in an area of $500 \times 500 \mathrm{~m} 2$ as shown below.

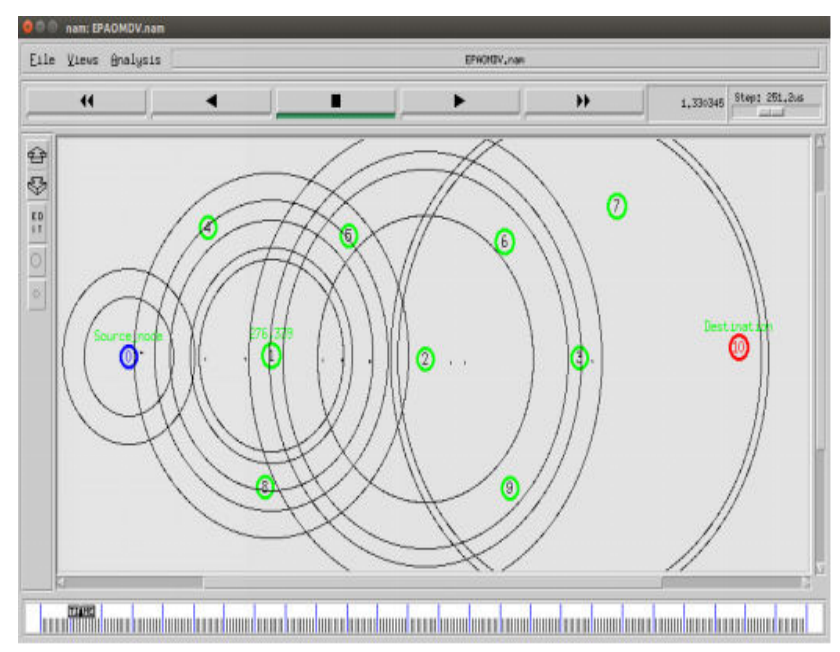

Fig 5: The working Simulation scenario of our protocol

The other simulation parameters that we used in our work are summarized in table below. We tested two cases: Efficient Path AOMDV i.e. EPAOMDV and AOMDV using fixed /static network topology as shown below. All the parameters used and the values assigned for those metrics in our simulation scenario are indicated in the Table 3 described below.

Table 3: Simulation parameter and their assigned values

\begin{tabular}{|c|l|l|}
\hline Para. No & Parameter type & \multicolumn{1}{|c|}{ Assigned values } \\
\hline 1 & Topology & Fixed \\
\hline 2 & Area $\left(\mathrm{m}^{2}\right)$ & $500 \mathrm{X} 500$ \\
\hline 3 & Channel type & Channel/Wireless channel \\
\hline 4 & Maximum Speed(m/sec) & 5 \\
\hline 5 & MAC type & MAC/802_11 \\
\hline 6 & Routing Protocol & AOMDV, EP-AOMDV \\
\hline 7 & No of Nodes & $16,20,30,50$ \\
\hline 8 & Antenna model & Antenna/omniAntenna \\
\hline 9 & Simulation time & $45 \mathrm{~s}$ \\
\hline 10 & radio-propagation mode & Propagation/TwoRayGround \\
\hline 11 & Packet Size(byte) & 1500 \\
\hline 12 & Transmission range & $250 \mathrm{~m}$ \\
\hline
\end{tabular}

\section{[6] Performance Evaluation Metrics AND RESULTS}

These are metrics that are used for evaluating our work. In our system we propose different performance evaluation metrics such as throughput including average throughput, packet drop rate, End to end delay and packet delivery ratio.

\section{[6.1] Throughput performance metric}

Throughput is a total number of delivered data packets divided by the total duration of the simulation time. In our case we evaluate throughput in terms of packets delivered per second. We calculate this metrics by storing the number of packet received by the sink node. The number of packet received is multiplied by 8 , divided by sample time and then divided by 1000 which achieve the kilo-bits per second [23].

Figure 6 illustrates the comparison of average throughput for EPAOMDV and AOMDV. In this metrics the throughput of the protocol in terms of the number of Message delivered per one second or kilo-bits per second is analyzed.

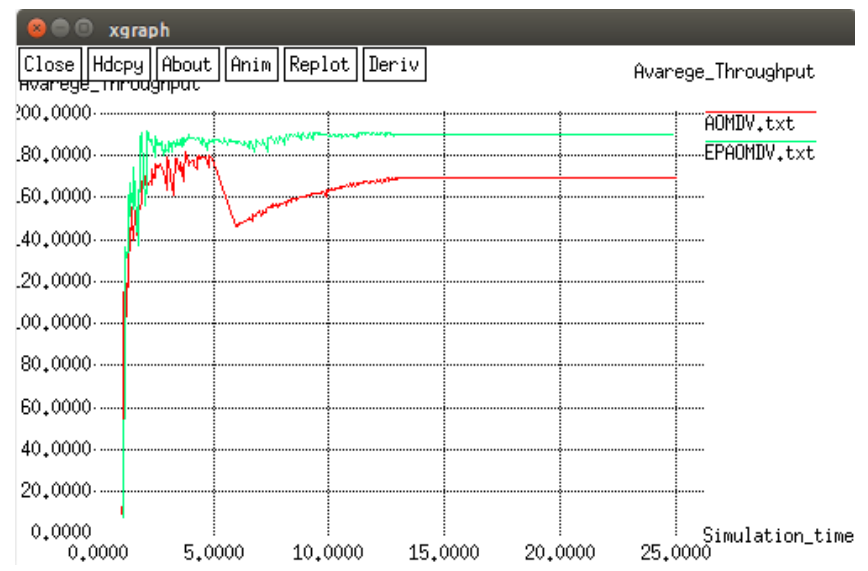

Fig 6: Average throughput comparison Vs simulation time In Figure below we illustrated the comparison of average 
throughput for EPAOMDV and AOMDV taking different simulation scenario or different node densities.

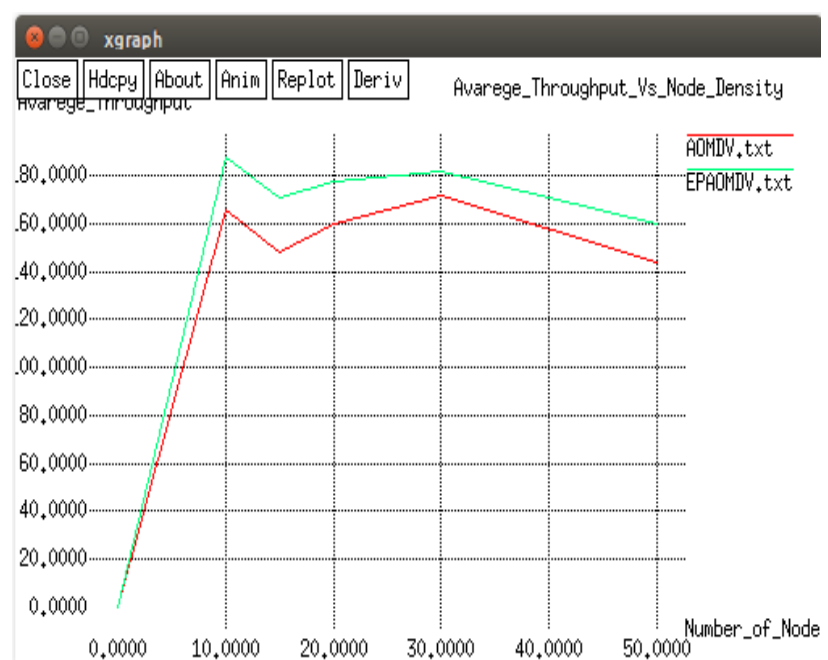

Fig 7: Average throughput comparison Vs node density

As the simulation results in Figure 6 and Figure 7 shows our EPAOMDV seem to achieve better performance than original AOMDV in terms of average throughput when we compare with their result.

\section{[6.2] Packets Drop ratio performance metric}

The term packet loss occurs when one or more packets of data traveling across a network fail to reach their destination. Packet loss is measured as a percentage of packets lost with respect to packets sent [24]. The packet drop rate is calculated first by storing the number of packets lost by the sink. The trace file provides the packets lost and all the other information. We then take this value and divide by the sample time to achieve packet loss per unit time or packet drop rate.

In AOMDV when the time of simulation increases the number of packets dropped also increases i.e. number of packets not successfully reaching the destination is very high as shown in Figure below. The EPAOMDV performs consistently well with increase in time of simulation.

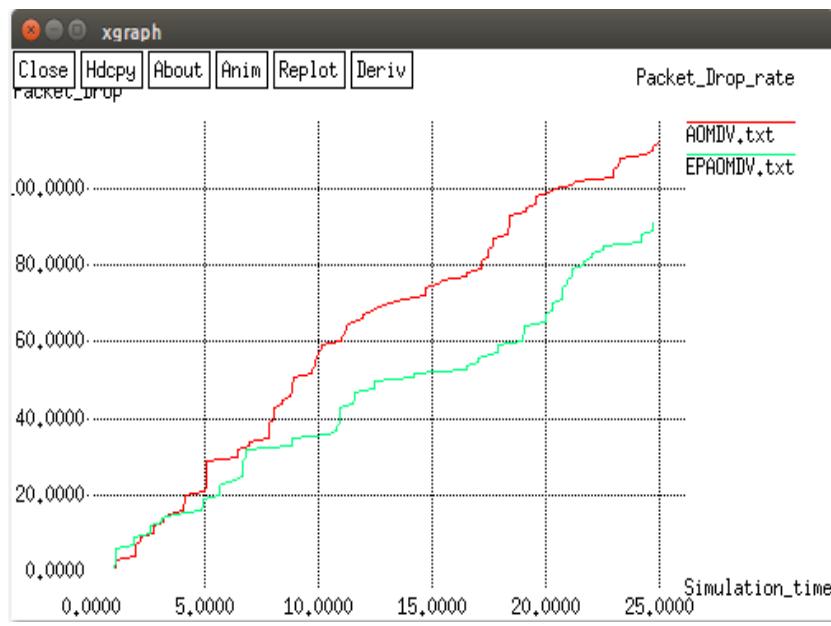

Fig 8: Packet drop comparison Vs simulation time

In Figure below we illustrated the comparison of average packet drop rate for EPAOMDV and AOMDV taking different simulation scenario or different node densities. As indicated on Figure 9, our scheme has less packet drop rate with increase in the number of nodes.

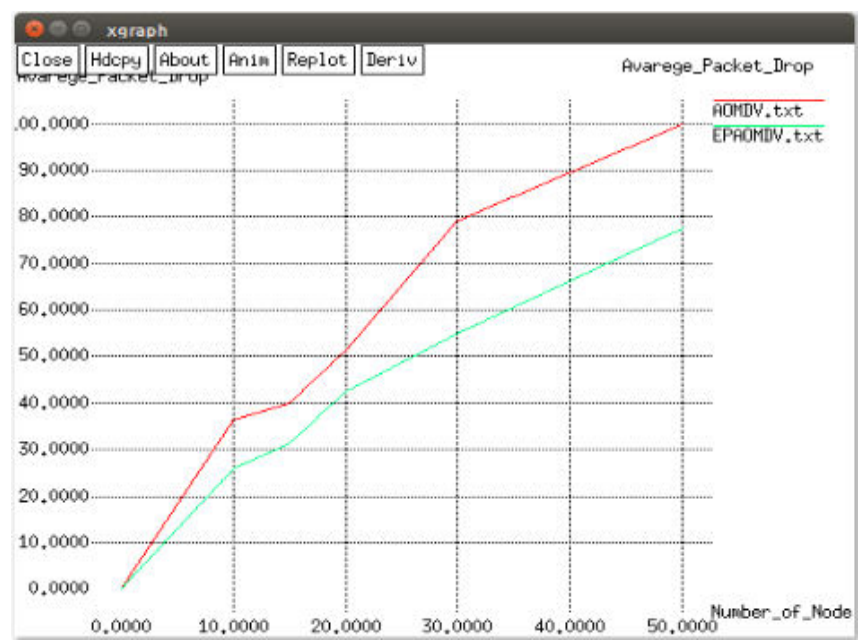

Fig 9: Packet drop comparison Vs node density

\section{[6.3] End to End delay performance metric}

End-to-End Delay is the interval time between sending by the source node and receiving by the destination node, which includes the processing time and queuing time. Average delay is the time taken to successfully transmit a packet from the sending node to the intended destination node [24].

Thus, for delay performance comparison purpose we use the average end to end routing delay of both protocols. Here figure below illustrates the comparison of average end to end delay for EPAOMDV and AOMDV taking different simulation scenario or different node densities as evaluation metrics.

As the figure indicates our proposed approach, EPAOMDV seems to deliver the packet faster than the original AOMDV protocol.

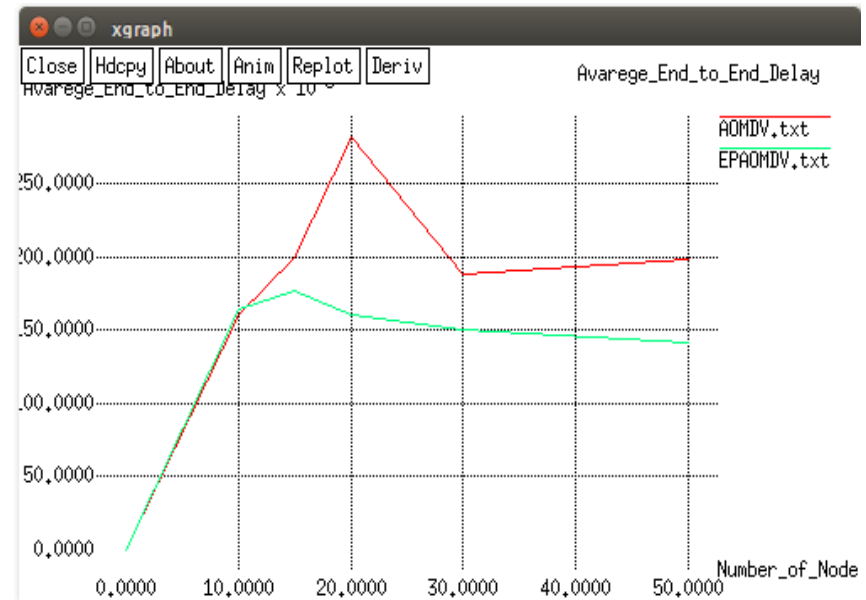

Fig 10: Average End to End delay Vs node density 
[6.4] Packets Delivery ratio performance metric Packets Delivery ratio is calculated as the ratio of packets received by the Sink node to the number of packets that are generated by the source nodes [24].

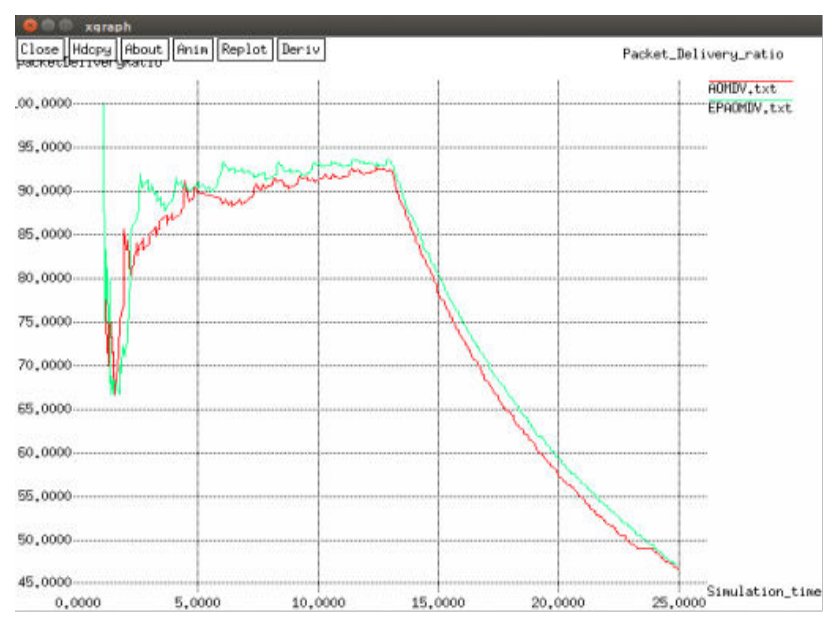

Fig 11: Packet Delivery Ratio Vs simulation time

The proposed efficient path selection mechanism has improved success rate when we compare with that of the hop count based path selection mechanism. The comparison is shown in Figure 11 and Figure 12.

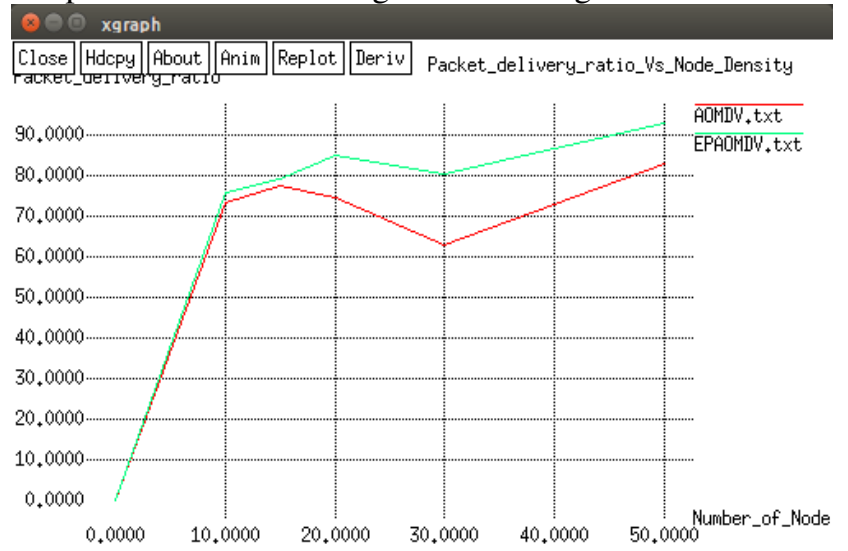

Fig 12: Packet Delivery Ratio comparison Vs node density

\section{[7] CONCLUSION}

In this thesis work, we have analyzed effect of the combined metrics: packet travel time, position of nodes, the nodes energy factor and the hop count in AOMDV routing protocols of MANET especially in emergency applications like medical alert system. Dealing with this all we have proposed an efficient path selection scheme for those kinds of applications using AOMDV routing protocol. To do so we have integrated the combined metrics of packet travel time, distance, the node energy factor and the hop counts into the original AOMDV protocol so that they all when combined will enable us to choose an efficient path with throughput guarantee, less delay and timely data delivery as it is indicated in our comparison graphs.
As a future work other parameters with change in the status of nodes in terms of packet dropping behavior and speed of nodes could be tested and considered.

\section{REFERENCES}

[1] Juan P. Macas, Recent Developments in Mobile Communications A Multidisciplinary Approach, ISBN 978-953-307-910-3, 2011 InTech

[2] Rajan Bansal, et al, Analytical Study the Performance Evaluation of Mobile Ad Hoc Networks using AODV Protocol, International Journal of Computer Applications (09758887), Volume 14 No.4, January 2011

[3] Pooja dahiya, Gunjan madan, Reema gupta, performance evaluation of AODV and AOMDV on the basis of throughput, IJCSMC, Vol. 3, Issue. 9, September 2014, pg. 277283

[4] Crawley, E., A Framework of QoS Based Routing in the Internet, RFC 2386,

[5] Lei Chen and Wendi B. Heinzelma, A Survey of Routing Protocols that Support QoS in Mobile Ad Hoc Networks, IEEE Network, Nov/Dec 2007

[6] K. Mounagurusamy and K. Eswaramurthy, A Novel Load Balancing Scheme for Multipath Routing Protocol in MANET, CIT. Journal of Computing and Information Technology, Vol. 24, No. 3, September 2016, 209220209 doi:10.20532/cit.2016.1002497.

[7] Tripti Sharma1 AND Dr. Vivek Kumar, Bandwidth Aware On Demand Multipath Routing In Manets, International Journal OF Wireless and Mobile Networks (Ijwmn) Vol. 6, No.4, 2014

[8] May Cho Aye, Aye Moe Aung, Energy Efficient Routing for MANETs using On demand Multipath Routing Protocol, International Journal of Advanced Research in Computer Engineering and Technology (IJARCET) Volume 3 Issue 5, May 2014

[9] T.Sivaraman, Dr. E. Karthikeyan, EE-BWA-AOMDV: Energy Efficient and Bandwidth Aware On-demand Multipath Routing protocol for Mobile Ad hoc Networks, International Journal of Computer Application (22501797) Volume 6 No.2, March- April 2016

[10] M.Sharma1, A. Brar, Enhancement in AOMDV Protocol to Reduce Chances of Link Failure in Mobile Adhoc Network, International Journal of Advanced Research in Computer Engineering and Technology (IJARCET) Volume 4 Issue 6, June 2015

[11] M. Abdulkader, S. Abdulkader, Load aware and Energy efficient approach to improve network connectivity in MANET, Faculty of engineering and technology Sam Higgin bottom university of agriculture, technology and sciences Allahabad, 211007, 2017

[12] Mohamed A. Ryan, Sayed Nouh, Tarek M. Salem, Abdelhady M. Naguib, EDA-AODV: Energy and Distance Aware AODV Routing Protocol, Volume 5, Issue 5, September October (2018)

[13] https://en.wikipedia.org/wiki/Transmission time 
[14] https://en.wikipedia.org/wiki/Distance

[15] Dr. M. Kokilamani, Dr. E. Karthikeyan, Video Streaming Evaluation of Path Efficient and Geographical Distance based AOMDV (PE-GD-AOMDV) Routing Protocol in MANET, International Journal of Advanced Networking and Applications, 10(5), 2019, 0975-0290

[16] Imrich Chlamtac, et al, Mobile Adhoc networking: imperatives and challenge Ad Hoc Networks 1 (2003) 1364, doi: 10.1016 / S1570-8705 (03) 00013-1, 2003 Elsevier B.V

[17] Pratik Singh, et.al, Flood Tolerant AODV Protocol FT-AODV, International Journal of Computer Applications (09758887), Volume 53 No.6, September 2012

[18] Dr. Shuchita Upadhayaya and Charu Gandhi, A Fault Tolerant Multipath routing protocol: International Journal of Wireless and Mobile Networks (IJWMN), Vol1, No 2, November 2009

[19] B. Stefano, C. Marco, G. Silvia and S. Ivan, Mobile Adhoc Network, IEEE Press, 2004.

[20] The VINT Project, The ns Manual, [Access date: Feb 2013

[21] Saba Siraj, et al, Network Simulation Tools Survey, International Journal of Advanced Research in Computer and Communication Engineering, Vol.1, Issue4,June2012

[22] Pratik Singh, et.al, Flood Tolerant AODV Protocol (FT-AODV, International Journal of Computer Applications (0975 8887), Volume 53 No.6, September 2012

[23] https://en.wikipedia.org/wiki/ Measuring network throughput

[24] Kurose, J. F, Ross, K.W. (2010). Computer Networking: A Top-Down Approach. New York: AddisonWesley.p.36. 